\title{
DIFFERENTIABILITY OF ENTROPY FOR ANOSOV AND GEODESIC FLOWS
}

\author{
A. KATOK, G. KNIEPER, M. POLLICOTT, AND H. WEISS
}

\section{INTRODUCTION}

Topological and metric entropies are among the most important global invariants of smooth dynamical systems. Topological entropy characterizes the total exponential complexity of the orbit structure with a single number. Metric entropy with respect to an invariant measure gives the exponential growth rate of the statistically significant orbits. The knowledge of entropies, especially in low-dimensional cases, provides a wealth of quantitative structural information about the system. Such information includes the growth rate of the number of periodic orbits [K 1], "large horseshoes" [K 3], the growth rate of the volume of cells of various dimensions ([Mn] for geodesic flows, $[\mathrm{Y}]$ in general), ergodic components, and factors with very stochastic behavior [Pe, Si], etc.

Unfortunately, because the entropies are defined in global asymptotic terms, a priori, we do not expect them to change smoothly when the system is perturbed, even in a very nice topology like $C^{\infty}$ or $C^{\omega}$ (real analytic).

There are several results concerning the regularity of entropy for general diffeomorphisms and flows on compact manifolds. Misiurewicz [Mi 1] constructed examples to show that the topological entropy, $h_{\text {top }}$ : $\operatorname{Diff}_{\infty}\left(M^{n}\right) \rightarrow \mathbf{R}$ is not continuous for $n \geq 4$. It seems unknown, although unlikely, whether entropy is continuous for $n=3$. Yomdin [Y] and Newhouse [N] have proved that $h_{\text {top }}: \operatorname{Diff}_{\infty}\left(M^{n}\right) \rightarrow \mathbf{R}$ is upper-semicontinuous for $n \geq 2$. Katok $[\mathrm{K} 1, \mathrm{~K} 3]$ has shown that for surfaces, $h_{\text {top }}: \operatorname{Diff}_{2}\left(M^{2}\right) \rightarrow \mathbf{R}$ is lower-semicontinuous. By combining these two results, one sees

\footnotetext{
Received by the editors April 10, 1989 and, in revised form, September 19, 1989.

1980 Mathematics Subject Classification (1985 Revision). Primary 58F15; Secondary $58 \mathrm{~F} 17$.

The first author was partially supported by NSF Grant \#DMS85-14630.

The fourth author is a Chaim Weizmann Research Fellow.
} 
that $h_{\text {top }}: \operatorname{Diff}_{\infty}\left(M^{2}\right) \rightarrow \mathbf{R}$ is continuous. This result also holds for $C^{\infty}$ flows on three-dimensional manifolds.

Misiurewicz [Mi 2] and Margulis (see [Y] for details) also proved that $h_{\text {top }}$ : $\operatorname{Diff}_{r}\left(M^{n}\right) \rightarrow \mathbf{R}$ is not upper-semicontinuous in the $C^{r}$ topology ( $r$ finite) for $n \geq 2$. Misiurewicz [Mi 2] has shown that for general $C^{k}$ flows on $M^{n}, k<\infty$ and $n \geq 3, h_{\text {top }}$ need not be continuous. It is unknown whether $h_{\text {top }}: \operatorname{Diff}_{r}\left(M^{n}\right) \rightarrow$ $\mathbf{R}$ is upper-semicontinuous on a residual set for $n \geq 3$, or whether $h_{\text {top }}: \operatorname{Diff}_{\infty}\left(M^{n}\right) \rightarrow \mathbf{R}$ is continuous on a residual set for $n \geq 3$.

For uniformly hyperbolic dynamical systems (i.e., Axiom- $A$ and Anosov diffeomorphisms and flows), the global orbit structure is stable under small perturbations. This easily implies that the topological entropy and the metric entropies with respect to natural invariant measures such as Liouville measure in the Hamiltonian case, Bowen-Ruelle measure for attractors, etc., change Hölder continuously.

The aim of this paper is to establish higher regularity results for the entropies of uniformly hyperbolic systems. Since the entropies determine, in a profound way, large elements in the global orbit picture of the system (see paragraph one), these elements change smoothly. We also deduce that the Bowen-Margulis measure changes smoothly in the weak topology. Our results set the stage for identifying some particularly remarkable ("rigid") dynamical systems by looking at critical points, extremal values, etc. for the entropy functions. In this direction, we obtain convenient formulas for the first derivatives for the topological and Liouville entropies in the case of geodesic flows on negatively curved manifolds. This enables us to characterize the critical points for the topological entropy for negatively curved surfaces. For real analytic systems, the holomorphic structures associated with the entropies provide new and potentially very useful invariants of the orbit structure.

We state our results for Anosov systems. Those results which are not specific to geodesic flows can be extended in a straightforward way to the more general case of Axiom- $A$ systems. To simplify notations, we talk about differentiability and analyticity along one parameter families, i.e., in the Gateaux sense. In fact, all our results carry over to Banach manifolds of maps and yield differentiability and analyticity in the Frechet sense. 


\section{BACKGROUND ON ANOSOV SYSTEMS}

Let $M$ be a compact $C^{\infty}$ Riemannian manifold and let $\phi^{t}: M \rightarrow M$ be a $C^{k}$ flow $(k \geq 1)$. We call this flow Anosov if there is a continuous splitting $T M=E^{0} \oplus E^{s} \oplus E^{u}$ into $D_{\phi}$ invariant subbundles such that:

(a) $E^{0}$ is the one-dimensional distribution tangent to the flow.

(b) There exist $C, \lambda>0$ such that for $t>0$ :

$$
\left\|D \phi^{t}(v)\right\| \leq C e^{-\lambda t}\|v\|, \quad v \in E^{s}
$$

and

$$
\left\|D \phi^{t}(v)\right\| \geq C e^{-\lambda t}\|v\|, \quad v \in E^{u} .
$$

The most important examples of Anosov flows are the geodesic flows for manifolds with negative sectional curvature [A].

Anosov systems are structurally stable and form an open subset in the $C^{1}$ topology of flows. They possess a unique measure of maximal entropy usually called the Bowen-Margulis measure. Margulis [Ma] and Bowen [B 2] have shown that the topological entropy of an Anosov flow, $h_{\text {top }}(\phi)$, has the following realization: Let $P(T) \equiv$ number of closed trajectories of $\phi^{t}$ with (prime) period $\leq T$. Then $h_{\text {top }}(\phi)=\operatorname{limit}_{T \rightarrow \infty}(1 / T) \log P(T)$. The subbundles $E^{s}$ and $E^{u}$ are uniquely integrable but in general they are only Hölder continuous. Since the integral manifolds of these subbundles (stable and unstable manifolds) profoundly determine the dynamical behavior, the main challenge in obtaining regularity results is to bypass the lack of regularity for the foliation structure.

\section{SMOOTHNESS OF TOPOLOGICAL ENTROPY}

Theorem 1 [KKPW]. Let $M$ be a compact $n$-dimensional manifold and let $\left\{\phi_{\lambda}^{t}\right\},-\varepsilon \leq \lambda \leq \varepsilon$ ( $\varepsilon$ sufficiently small) be a $C^{\omega}$ (real analytic) perturbation of a $C^{\omega}$ Anosov flow $\phi^{t} \equiv \phi_{0}^{t}$. Then $h_{\text {top }}\left(\phi_{\lambda}^{1}\right)$ is $C^{\omega}$.

We give two proofs of Theorem 1. The first proof uses zeta functions and complex analysis and only works for $C^{\omega}$ perturbations. This proof yields valuable insight into how the periods of closed trajectories change when the flow is perturbed. We also believe that the techniques in this proof may lead to advances in the study of qualitative properties of holomorphic dynamical systems.

Sketch of Proof. If $\phi^{t}$ is an Anosov flow on $M$, we define $d(s) \equiv$ $\prod_{\tau}(1-\exp (-s l(\tau)))$, where $l(\tau)$ is the least period of the closed 
orbit $\tau, s$ is a complex variable, and the Euler product is over all closed orbits. $d(s)$ is the reciprocal of the zeta function associated to $\phi^{t}$. It is easy to prove that $d(s)$ defines a nonvanishing holomorphic function for $\mathrm{re}(s)>h_{\mathrm{top}}(\phi)$ [PP]. Pollicott [Po], following ideas of Ruelle [R], has shown that $d(s)$ has a holomorphic continuation into a slightly larger half plane with a zero at $s=h_{\text {top }}(\phi)$. To study the regularity of topological entropy under perturbations, we study how the zeros of $d(s)$ vary. The proof has four main steps:

Step 1 . Show that $d(\lambda, s)$ is real analytic in $\lambda$ for $\operatorname{re}(s)$ sufficiently large. We need to study the function $\lambda \rightarrow l_{\lambda}(\tau)$, i.e., the function which assigns to each $\lambda$ the least period of the closed trajectory of $\phi_{\lambda}^{t}$ corresponding (under structural stability) to the closed trajectory $\tau$ of $\phi_{0}^{t}$. It is easy to show that for each fixed $\tau$, this function is real analytic, and hence has a holomorphic extension to a neighborhood of $(-\varepsilon, \varepsilon)$. We use symbolic dynamics to find an open set $V$ in $\mathbf{C}$ which contains $(-\varepsilon, \varepsilon)$ on which all least period functions have holomorphic extensions. This allows us to prove that the Euler product converges uniformly and hence defines a holomorphic function in $V$. The restriction of $d(\lambda, s)$ to $V \cap(-\varepsilon, \varepsilon)$ is real analytic.

Step 2. For each $\lambda \in(-\varepsilon, \varepsilon)$, apply Pollicott's result to holomorphically continue $d(\lambda, s)$ to a larger half plane. Care must be taken to ensure uniformity of the extension. We denote the extended function $\bar{d}(\lambda, s)$.

Step 3. Show that $\bar{d}(\lambda, s)$ is real analytic in $\lambda$. This step requires a deep result of Bernard Shiffman [Sh] on the dependence of analytic continuation on an external parameter.

Step 4 . Conclude that the zeros $h_{\text {top }}(\lambda)$ of $\bar{d}(\lambda, s)$ vary real analytically in $\lambda$.

Theorem 2 [KKPW]. Let $M$ be a compact $n$-dimensional manifold and let $\left\{\phi_{k}^{t}\right\},-\varepsilon \leq \lambda \leq \varepsilon$ ( $\varepsilon$ sufficiently small) be a $C^{k+1}$ perturbation of a $C^{k+1}$ Anosov flow $\phi^{t} \equiv \phi_{0}^{t}, 1 \leq k \leq \infty$. Then $h_{\text {top }}\left(\phi_{\lambda}^{1}\right)$ is $C^{k}$.

Sketch of proof. The proof of Theorem 2 also works in the $C^{\omega}$ case, and gives a second proof of Theorem 1. We first use Markov partitions [B 1] to reduce the problem to symbolic flows. Our strategy, which was influenced by the work of Ruelle, is to show that the map $(\lambda, x) \mapsto P\left(-x r_{\lambda}\right)$ is $C^{k}$, where $r_{\lambda}$ denotes the 
return map between sections for $\phi_{\lambda}^{t}$ (the height function over $\Sigma_{A}$ ), and $P(f)$ denotes the pressure of $f$. It is easy to show that $P\left(-x r_{\lambda}\right)=0$ implies $x=h_{\text {top }}(\lambda)$. We then apply the Implicit Function Theorem to conclude that $h_{\text {top }}(\lambda)$ is $C^{k}$.

Step 1 . Show that the map $C^{\alpha}\left(\Sigma_{A}\right) \rightarrow \mathbf{R}$ defined by $f \mapsto P(f)$ is analytic [R].

Step 2. Show that the map $(-\varepsilon, \varepsilon) \rightarrow C^{\alpha}\left(\Sigma_{A}\right)$ defined by $\lambda \mapsto r_{\lambda}$ is $C^{k}$. This entails showing that the maps obtained from structural stability depend $C^{k}$ on the perturbation parameter in the $C^{r}$ topology of maps. The proof of this result also uses the Implicit Function Theorem: The nondegeneracy condition is that the graph transform induced by the time one map of an Anosov flow be hyperbolic on $C^{\alpha}$ sections of the tangent bundle.

In Theorem 2, we require a $C^{2}$ perturbation of a $C^{2}$ Anosov flow to ensure that the topological entropy changes in a $C^{1}$ way. Katok, Knieper, and Weiss [KKW], using different methods, prove a stronger result. They show that a $C^{1}$ perturbation results in a $C^{1}$ change in entropy. They also find the following useful formula for the first derivative of topological entropy:

Theorem $3[\mathrm{KKW}]$. Let $\phi^{t}$ be a $C^{1}$ Anosov flow on a compact $n$ dimensional manifold $M$ and let $\left\{\phi_{\lambda}^{t}\right\},-\varepsilon<\lambda<\varepsilon$ ( $\varepsilon$ sufficiently small ) be a $C^{1}$ perturbation of $\phi^{t} \equiv \phi_{0}^{t}$. Then $h_{\mathrm{top}}\left(\phi_{\lambda}^{1}\right)$ is $C^{1}$ and

$$
\left.\frac{\partial h_{\text {top }}}{\partial \lambda}\right|_{\lambda=0}=\left.h_{\text {top }}(0) \int_{M} \frac{\partial \alpha_{\lambda}(p)}{\partial \lambda}\right|_{\lambda=0} d \mu_{0}^{B M},
$$

where $\mu_{0}^{B M}$ denotes the Bowen-Margulis measure (the measure of maximal entropy) for $\phi^{t}$ and $\alpha_{\lambda}(p)$ denotes any function which, when integrated along orbits of $\phi^{t}$, gives the time reparametrization in structural stability.

Theorem 4 [KKW]. Let $\left(M^{n}, g\right)$ be a compact Riemannian manifold of negative sectional curvature, and let $\left\{g_{\lambda}\right\},-\varepsilon<\lambda<\varepsilon$ ( $\varepsilon$ sufficiently small) be a $C^{2}$ perturbation of $g \equiv g_{0}$. If $\phi_{\lambda}^{t}$ denotes the geodesic flow associated to $g_{\lambda}$, then $h_{\text {top }}\left(\phi_{\lambda}^{1}\right)$ is $C^{1}$ and

$$
\left.\frac{\partial h_{\mathrm{top}}(\lambda)}{\partial \lambda}\right|_{\lambda=0}=\left.\frac{-h_{\mathrm{top}}(0)}{2} \int_{S M_{0}} \frac{\partial g_{\lambda}(v, v)}{\partial \lambda}\right|_{\lambda=0} d \mu_{0}^{B M},
$$

where $\mu_{0}^{B M}$ denotes the Bowen-Margulis measure (the measure of maximal entropy) for $\phi^{t}$. 
The proofs of Theorems 3 and 4 are based on obtaining a good estimate of how the periods of most closed orbits change when you perturb the flow, most in the sense of enough to give topological entropy. The estimate follows from the methods which the first author developed in [K 1] and [K 2].

The following result on the critical points of topological entropy is an application of Theorem 4 .

Theorem 5 [KKW]. Let $\mathscr{R}_{1}\left(M^{2}\right)$ denote the submanifold of negatively curved metrics on a compact surface $M$ having area equal to 1. Then $h_{\text {top }}: \mathscr{R}_{1}\left(M^{2}\right) \rightarrow \mathbf{R}$ has a critical point at $g \in \mathscr{R}_{1}\left(M^{2}\right)$ if and only if $g$ is a metric of constant negative curvature.

Theorem 6 [KKW]. Let $\left(M^{n}, g\right)$ be a compact $n$-dimensional Riemannian manifold with no conjugate points, and let $\left\{g_{\lambda}\right\},-\varepsilon<$ $\lambda<\varepsilon$ ( $\varepsilon$ sufficiently small) be a $C^{2}$ perturbation of $g=g_{0}$. Then $h_{\text {top }}\left(\phi_{\lambda}^{1}\right)$ is Lipschitz.

The proof of Theorem 6 is based on Manning's formula [Mn] and a simple geometric estimate.

SMOothNeSS OF ENTROPy, PRESSURE, AND GibBS MEASURES

Let $M$ be a compact $n$-dimensional manifold and let $\left\{\phi_{\lambda}^{t}\right\}$, $-\varepsilon \leq \lambda \leq \varepsilon$ ( $\varepsilon$ sufficiently small) be a $C^{k+1}$ perturbation of a $C^{k+1}$ Anosov flow $\phi^{t} \equiv \phi_{0}^{t}$. Let $\left\{H_{\lambda}\right\}$ be a family of Hölder homeomorphisms which is $C^{k}$ in $\lambda$ and which realizes the orbit equivalence between $\phi_{\lambda}^{t}$ and $\phi^{t}$. The following rather technical result provides a useful generalization of Theorems 1 and 2 .

Theorem 7. Let $\left\{f_{\lambda}\right\}$ be a family of Hölder continuous functions such that the map $(-\varepsilon, \varepsilon) \rightarrow C^{\alpha}\left(M^{n}\right)$ defined by $\lambda \rightarrow f_{\lambda} \circ H_{\lambda}$ is $C^{k}$. Then the pressure function $\lambda \rightarrow P\left(f_{\lambda}, \phi_{\lambda}^{1}\right)$ is $C^{k}$. The same holds for real analytic families.

Corollary 1. Let $f \in C^{k+1}\left(M^{n}\right)$. Then the pressure function $\lambda \rightarrow$ $P\left(f, \phi_{\lambda}^{1}\right)$ is $C^{k}$.

Corollary 2 (Gibbs measures are weakly smooth). For $C^{k+1}$ families of Anosov flows $\left\{\phi_{\lambda}^{t}\right\}$ and fixed $\rho \in C^{k+1}\left(M^{n}\right)$, the map $(-\varepsilon, \varepsilon) \times C^{k+1}\left(M^{n}\right) \rightarrow \mathbf{R}$ defined by $(\lambda, f) \rightarrow \int_{M} \rho d \mu_{\lambda, f}$ is $C^{k-1}$, where $\mu_{\lambda, f}$ denotes the $\phi_{\lambda}^{t}$ Gibbs state for $f$. 
Corollary 3 (Smoothness of entropy for Gibbs states). For $C^{k+1}$ families of Anosov flows $\left\{\phi_{\lambda}^{t}\right\}$ and fixed $f \in C^{k+1}\left(M^{n}\right)$, the function $\lambda \rightarrow h_{\mu_{\lambda, f}}$ is $C^{k-1}$, where $h_{\mu_{\lambda, f}}$ denotes the measure theoretic entropy with respect to the $\phi_{\lambda}^{t}$ Gibbs state for $f$.

\section{SMOOTHNESS OF METRIC ENTROPY FOR LIOUVILLE MEASURE}

Knieper and Weiss have investigated the regularity of the measure theoretic entropy $h_{\mu}$ for $C^{k}$ perturbations of geodesic flows and Anosov flows which possess a smooth invariant measure $\mu$. The main result [KW 2] combines a parametric version of the Hirsch-Pugh-Shub $C^{r, \alpha}$ Theorem [HP 1] along with Sinai's entropy formula to show that metric entropy possesses at least as much smoothness as the individual stable and unstable foliations, as long as the smoothness follows from a global contraction theorem (graph transform). If we combine this result with results of Hirsch and Pugh [HP 2] on the smoothness of the foliations for geodesic flows in negative curvature, we obtain the following theorem on the smoothness of metric entropy with respect to Liouville measure:

\section{Theorem 8 [KW 2].}

(i) Let $\left(M^{2}, g\right)$ be a compact negatively curved surface, and let $\left\{g_{\lambda}\right\},-\varepsilon<\lambda<\varepsilon$ ( $\varepsilon$ sufficiently small) be a $C^{3}$ perturbation of $g \equiv g_{0}$. Then $h_{\mu_{i}}\left(\phi_{\lambda}^{1}\right)$ is $C^{1, \alpha}$ for all $\alpha<1$.

(ii) Let $\left(M^{n}, g\right)$ be a compact $n$-dimensional manifold with negative sectional curvature $-4<K \leq-1$, and let $\left\{g_{\lambda}\right\}$, $-\varepsilon<\lambda<\varepsilon$ be a $C^{3}$ perturbation of $g \equiv g_{0}$ through metrics having negative sectional curvature $-4<K_{\lambda} \leq-1$. Then $h_{\mu_{\lambda}}\left(\phi_{\lambda}^{1}\right)$ is $C^{1, \alpha}$, where $\alpha=\alpha\left(g, g_{\lambda}\right)$.

The following theorem was a precursor to Theorem 8 .

Theorem [KW 1]. Let $\left(M^{2}, g\right)$ be a compact negatively curved surface, and let $\left\{g_{\lambda}\right\},-\varepsilon<\lambda<\varepsilon$ (e sufficiently small) be a $C^{3}$ perturbation of $g \equiv g_{0}$. Then $h_{\mu_{i}}\left(\phi_{\lambda}^{1}\right)$ is $C^{1}$, where $h_{\mu_{j}}\left(\phi_{\lambda}^{1}\right)$ denotes the measure theoretic entropy of the $g_{\lambda}$ geodesic flow with respect to Liouville measure.

We give a very geometric proof of this result. We use Pesin's entropy formula to reduce the problem to studying how the nor- 
mal curvature of the stable and unstable horocycles orthogonal to a fixed unit tangent vector changes when the metric is smoothly perturbed. We consider the corresponding question for circles of radius $r$ orthogonal to a fixed unit tangent vector and let $r \uparrow \infty$. We then justify several limiting operations. Along the way, we obtain an integral formula for the derivative.

Gerhard Knieper has recently proved that measure theoretic entropy (with respect to Liouville measure) is twice differentiable at negatively curved locally symmetric spaces, and he has an explicit formula for the second derivative at constant negatively curved metrics.

\section{Theorem 9 [Kn].}

(i) Let $\left(M^{n}, g\right)$ be a compact locally symmetric space with negative sectional curvature and let $\left\{g_{\lambda}\right\}$ be a $C^{\infty}$ perturbation of $g \equiv g_{0}$. Then $h_{\mu_{j}}\left(\phi_{\lambda}^{1}\right)$ is twice differentiable at $\lambda=0$.

(ii) Let $\left(M^{n}, g\right)$ be a metric of constant negative curvature $k_{0}$ and let $\left\{g_{\lambda}\right\}$ be a $C^{\infty}$ perturbation of $g \equiv g_{0}$ preserving the Riemannian volume. Furthermore, let $s(\lambda)$ denote the total scalar curvature of $g_{\lambda}$. Then

$$
\begin{aligned}
& \left.\frac{\partial^{2} h_{\mu_{\lambda}}\left(\phi_{\lambda}^{1}\right)}{\partial \lambda^{2}}\right|_{\lambda=0} \\
& \quad=-\frac{n-1}{\sqrt{k_{0}}}\left(\int_{S M} \operatorname{tr}\left(\left.\frac{\partial}{\partial \lambda}\right|_{\lambda=0} H_{\lambda}(v)\right)^{2} d \mu_{0}^{B M}+\frac{1}{2(n-1)} s^{\prime \prime}(0)\right),
\end{aligned}
$$

where $H_{\lambda}(v)$ denotes the second fundamental form of the unstable horosphere through $v$.

\section{REFERENCES}

[A] D. Anosov, Geodesic flows on closed Riemannian manifolds with negative curvature, Proc. Steklov Inst. Math. 90 (1967), 235.

[B 1] R. Bowen, Symbolic dynamics for hyperbolic flows, Amer. J. Math. 95 (1972), 429-459.

[B 2] _ Periodic orbits for hyperbolic flows, Amer. J. Math. 94 (1972), 1-30.

[HP 1] M. Hirsch and C. Pugh, Stable manifolds and hyperbolic sets, Proc. Sympos. Pure Math. XIV (1968), 133-165.

[HP 2] _ Smoothness of horocycle foliations, J. Differential Geom. 10 (1975), $225-238$. 
[K 1] A. Katok, Lyapunov exponents, entropy and periodic orbits for diffeomorphisms, Publ. Math. Inst. Hautes Études Sci 51 (1980), 137-173.

[K 2] - Four applications of conformal equivalence to geometry and dynamics, Ergodic Theory Dynamical Systems Conley Memorial Issue $\mathbf{8}^{*}$ (1988), 139-152.

[K 3] - Nonuniform hyperbolicity and structure of smooth dynamical systems, Proc. of International Congress of Mathematicians 1983, Warszawa, 2, pp. 1245-1254.

[KKPW] A. Katok, G. Knieper, M. Pollicott, and H. Weiss, Differentiability and analyticity of topological entropy for Anosov and geodesic flows, Invent. Math., 98 (1989), 581-597.

[KKW] A. Katok, G. Knieper, and H. Weiss, Regularity of topological entropy for geodesic and Anosov flows, preprint.

[Kn] G. Knieper, On the second derivative of metric entropy at locally symmetric spaces, in preparation.

[KW 1 G. Knieper and H. Weiss] Differentiability of measure theoretic entropy: I, Invent. Math. 95 (1989), 579-589.

[KW 2] __ Smoothness of measure theoretic entropy for Anosov flows, preprint.

[Mn] A. Manning, Topological entropy for geodesic flows, Ann. of Math. 110 (1979), 567-573.

[Ma] G. Margulis, Applications of ergodic theory to the investigation of manifolds of negative curvature, Functional Anal. Appl. 3 (1969), 335-336.

[Mi 1] M. Misiurewicz, On non-continuity of topological entropy, Bull. Acad. Polon. Sci., Ser. Sci Math. Astro. Phys. 19 (4), (1971), 319-320.

[Mi 2] __ Diffeomorphisms without any measure with maximal entropy, Bull. Acad. Polon. Sci., Ser. Sci. Math. Astro. Phys. 21 (10), (1973), 903-910.

[N] S. Newhouse, Continuity properties of entropy, Ergodic Theory Dynamical Systems Conley Memorial Issue 8* $^{*}$ (1988), 283-300.

[PP] W. Parry and M. Pollicott, An analogue of the prime number theorem for closed orbits of Axiom A flows, Ann. of Math. 118 (1983), 573-592.

[Pe] Y. Pesin, Characteristic Lyapunov exponents and smooth ergodic theory, Russian Math. Surveys 32 (4), (1977), 55-114.

[Po] M. Pollicott, Meromorphic extensions of generalized zeta functions, Invent. Math. 85 (1986), 147-164.

[R] D. Ruelle, Thermodynamics formalism, Addison-Wesley, Reading, Mass., 1978.

[Sh] B. Shiffman, Separate analyticity and Hartogs theorems, preprint.

[Si] J. Sinai, On weak isomorphism of transformations with invariant measures, Math. USSR Sb. 63 1, (1964), 23-42.

[Y] Y. Yomdin, Volume growth and entropy, Israel J. Math. 57 (1987), 285-300.

California Institute of Technology, Pasadena California, 91125

Freie Universitaet Berlin, Arnimalle 3, 1000 Berlin 33

Centro de Mathematica da Universidade do Porto, Prasa Gomes Teixeiro, 4000 Porto, Portugal

California Institute of Technology, Pasadena, California 91125 
